\title{
The HIV and Sexual Reproductive Health Status of Young People in Swaziland: The Rationale for Focused Youth Investment
}

Bongani Robert Dlamini ${ }^{*}$, Phumzile Mabuza ${ }^{2}$, Zandile Masangane ${ }^{2}$, Thamary Silindza ${ }^{1}$, Makhosami Dlamini ${ }^{3}$ and Phumzile Dlamini ${ }^{1}$

${ }^{1}$ HIV and Youth, UNFPA, Swaziland

${ }^{2}$ Ministry of Health, Swaziland

${ }^{3}$ SNYC, Swaziland

\begin{abstract}
Background: Youth are the future, and investing in the youth of today means a brighter future for the entire world. Nowhere is this truer than in Africa, where changing demographics are creating a unique opportunity to harness the potential of young people to accelerate development. Swaziland, with a large and growing youth population and a declining birth rate, is on the cusp of a critical demographic transition.

Methods: The HIV and Sexual Reproductive Health Status of Young People in Swaziland analysis were prepared in stages: desk review and analysis, consultations/interview meetings with key stakeholders, data analysis and compilation of the report.

Results and discussion: Early sexual debut, high adolescent fertility rate, unmet need for family planning, and on-going problems with sexual and gender based violence (GBV) are some of the key issues faced by young people in terms of reproductive health in Swaziland. Adolescent fertility did decline slightly from 89/1000 to 87/1000 between 2010 and 2014. HIV prevalence is much higher in young women than young men across all age groups, and marked increases in prevalence become apparent throughout the 20s for both sexes. Approximately 1 in 4 females in Swaziland experienced physical violence as a child and 9\% of the youth aged 18-24 experienced coerced sexual intercourse before they turned eighteen.
\end{abstract}

Conclusion: There seems to be some improvement in sexuality education and behaviour change interventions. There is gradual increase in age at sexual debut and in the proportion of adolescents reporting to be abstaining from sexual activity. This suggests that some interventions may have dissuaded some adolescents from sexual intercourse.

Recommendations: A minimum standard for health facilities to be considered youth-friendly should be put in place, and all facilities should be assessed against the standard as well as linkages between schools, communities and health facilities should prioritized.

Keywords: HIV; Adolescents; Youth; Young people

\section{Introduction}

The world is home to over 1.8 billion young people between the ages of 10 and 24 years, with Africa having the youngest population in the world, with over 344 million estimated to be between the ages of 10 and 24 years, out of a total population of 1.2 billion [1]. In sub-Saharan Africa young people make up the greatest proportion of the population, with more than one-third of the population between the ages of 10 and 24 years - the only region in the world in which the number of young people continues to grow substantially [2]. Projections are that in 2025 the number of young people (aged 10 to 24) in sub-Saharan Africa is expected to increase to 436 million, and further increase to 605 million by 2050 [1,2]. Never before has the world, Africa and the Southern Africa region experienced such an unprecedented number of young people, and just these sheer numbers on their own are a strong call for accelerated efforts to effectively address their needs and challenges. Swaziland, like many other African states has a very youthful population, with about $34 \%$ of the population aged $10-24$ years, and this translates to over 400,000 young Swazis, out of a total population of about 1.2 million people [3].

HIV/AIDS is continuing to be one of the most pressing challenges that young people in Swaziland are facing, with heightened vulnerabilities for girls and young women. HIV prevalence among young females aged $15-19$ years stands at $10.2 \%$, compared to $1.9 \%$ for males of the same age. In the age group 20-24, HIV prevalence amongst females is $38.2 \%$, compared to $12.3 \%$ for males in the same age bracket.
HIV incidence is also significantly higher amongst young Swazi females (15-19) compared to the males same age group standing at: 3.84 for females compared to 0.84 for males [4].

Teenage pregnancy is another challenge for the young Swazi girls. Currently the adolescent birth rate in the country stands at 87 per 1000 adolescents, and teenage pregnancy is one of the main factors contributing to school dropouts in the country [5]. In addition, young people in Swaziland continue to experience very high levels of violence, again disproportionately affecting girls compared to boys. The national estimate is that 1 in 3 women had experienced some form of sexual violence by the time they were 18 years [6]. A very unfortunate statistic indeed, indicating that Swazi children experience sexual gender-based violence very early in their lives, at a time when all that should be occupying their minds is school and play. On the hand, very limited number of health care facilities in the country has youth-friendly

*Corresponding author: Bongani Robert Dlamini, Program Analyst-SRH, HIV and Youth, UNFPA, Swaziland; Tel: +26876176844; E-mail: bonganidlamini.bd@gmail.com

Received February 14, 2017; Accepted February 22, 2017; Published March 01, 2017

Citation: Dlamini BR, Mabuza P, Masangane Z, Silindza T, Dlamini M, et al. (2017) The HIV and Sexual Reproductive Health Status of Young People in Swaziland: The Rationale for Focused Youth Investment. J AIDS Clin Res 8: 669. doi: 10.4172/2155 6113.1000669

Copyright: (c) 2017 Dlamini BR, et al. This is an open-access article distributed under the terms of the Creative Commons Attribution License, which permits unrestricted use, distribution, and reproduction in any medium, provided the original author and source are credited. 
services, and this deters young people from accessing SRH information and services from these institutions [7]. The aim of this retrospective analysis is to provide a comprehensive and cross-sectoral picture of the HIV and Sexual Reproductive Health (SRH) Status of Young People in Swaziland by gathering data and documenting conditions that affect young people in the country.

\section{Methodology}

The HIV and Sexual Reproductive Health Status of Young People report in Swaziland was prepared in stages. Desk Review and Analysis involved collecting, analysing, and synthesizing background documents. Specific attention was focused on key policies affecting the various thematic areas and the programmatic interventions in place to support progress therein. Consultations/interview meetings with key stakeholders were undertaken in order to fully understand key policy framework issues and policy and programmatic gaps. A standard interview guide was used during the interviews. The standard guide had sections, which included demographic information (such qualifications, experience in HIV and SRH) of the respondents, status of HIV and Sexual Reproductive Health for youth in Swaziland, SRH and HIV programmes, and sources of information on HIV and SRH for young people in the country. The guide open-ended questions, which allowed respondents to give their own ideas of HIV and SRH in Swaziland. In addition, focus group discussions (FGDs) with youth were also conducted, to enrich the participatory nature of the report.

\section{Data Management and Analysis}

Data analysis and compilation of the report was accomplished by triangulating the information collected during the desk review, FGDs, and stakeholder consultations. In addition, demographic and statistical analyses were conducted, mainly using secondary data sources such as the population census of Swaziland, the Demographic and Health Survey (DHS) of 2007-2008, the interim DHS of 2012, the Multiple Indicator Cluster Survey (MICS) 2010 and preliminary findings from MICS 2014, and various sector-specific regular surveillance and evaluation publications, such as the Annual Education Census reports and various Ministry of Health annual programme reports. Stakeholder's workshops took place in four forms to discuss and vet the State of the Youth Report. First, an inception workshop took place prior to undertaking any research, to develop consensus and understanding on the methodology for preparing the report. Second, an input workshop was held after producing a draft report, to discuss and suggest areas within the report that could be improved. Third, a validation workshop was conducted to review the revised draft. Finally, a fourth editorial and review workshop was conducted to review and edit the report structure and strengthen the conclusions and recommendations.

\section{Results and Discussion}

\section{Background and policy environment}

Youth in Swaziland experience a broad range of sexual and reproductive health challenges. Particularly for youth within the younger age category of 15-24, have specific vulnerabilities, largely related to age: physiological vulnerability, high susceptibility to peer pressure, tendencies toward risk-taking behaviour, lower ability to negotiate safer sex practices, and difficulty accessing sexual and reproductive health information and services [8].

The SRHR challenges of Swaziland's youth are exacerbated by a variety of the contextual factors. There is a low level of progression from primary to secondary school, high unemployment rates among young people, and high levels of poverty. A lack of education puts the power of young people to make protective and informed decisions for themselves and the economic vulnerabilities associated with poverty and unemployment put young people at risk of engaging in behaviours that make them susceptible to the acquisition of HIV and other STIs [9]. Additionally, high levels of sexual abuse and gender-based violence affect young people especially.

Adolescents and youth in Swaziland do not have adequate information and accessibility to services which will enable them to make informed decisions on their sexuality and reproductive health [10]. The vulnerabilities that this creates for young people are especially critical to address, as young people engage in sexual activity at early ages, and with limited knowledge of sexual and reproductive health and rights, lack skills to negotiate safer sex, access appropriate health commodities, and exhibit poor health-seeking behaviour [11].

The policy environment for the provision of SRH and HIV services to young people is favourable. This is evident by the availability of various policies in support of provision of SRH and HIV services to the young people. The National Health Policy of 2007 emphasizes decentralization of services and access to appropriate care at community levels, which is critical for young people to access necessary healthcare [12]. The National Policy on Sexual and Reproductive Health of 2013 states that comprehensive sexuality education, information, and integrated SRH and HIV services shall be provided to children, adolescents, and youth people at all levels of health care delivery systems and other relevant settings according to their age and need [10]. The policy also stipulates that the Ministry of Health shall provide an enabling environment and resources to provide adolescent sexual reproductive health services and that quality family planning information and care shall be provided to all reproductive-age (defined as 15-49) women and men [10]. The National Youth Policy calls for improved access to HIV/AIDS treatment for youth, the integration of Life Skills Education (which includes sexuality education) curricula into all institutions, the promotion of school- and community-based health clubs, and scaleup of SRH services targeting all youth, to reduce STI prevalence and unplanned pregnancies [13]. All of these policies emphasize that heath care delivery should be appropriate to the age of youth being served.

Coordinated multi-sectoral interventions have also been put in place by the Government in partnership with stakeholders to improve the SRH status of youth and their access to SRH services, for example by implementing a Social and Behavioural Change Communication (SBCC) strategy or the HIV prevention toolkit, rollout of Life Skills Education (LSE) curriculum and training of health care workers on adolescent youth friendly health services. Community-based education through sports, arts, and cultural activities, are some mechanisms utilized to reach out-of-school youth. These forums are intended to increase the utilization of services on the part of young people by increasing their access to information on sexuality and sexual and reproductive health and rights. Government and various partners have also intensified efforts to provide Comprehensive Sexuality Education in school, under the LSE programme, and out-of-school, through various community-based activities focused on youth.

\section{Reproductive health}

Early sexual debut, high adolescent fertility rate, unmet need for family planning, and on-going problems with sexual and gender based violence (GBV) are some of the key issues faced by young people in terms of reproductive health. There are many indicators that highlight a need for a comprehensive sexuality education and evidence based interventions. 


\section{Early sexual debut}

Young people in Swaziland begin engaging in sexual activity at an early age. The median age at first intercourse in Swaziland is 16 years for girls and 18 years for boys, while the median age at first marriage is 24 years [4]. Delaying first sexual intercourse is strongly correlated with more years of education among young women, and a reduced risk to HIV exposure and early pregnancy [14].

Sexual activity increases with age and women aged 25-39 are the most sexually active. Worth noting is that nearly 3 in 5 women aged 1519 have never had sex, while 13 percent were sexually active at the time of the survey [15]. However, evidence suggest that close to $40 \%$ of young women under the age of 19 years have at some point engaged in sexual intercourse, which demonstrates a need for early and comprehensive SRH and HIV education and youth-friendly SRH services [11,14].

\section{Adolescent fertility rate, teenage pregnancy and early motherhood}

Teenage pregnancy in Swaziland remains a cause for concern. In SDHS 2007, teenage pregnancy was at $24 \%$ and over the years it has not shown responding decline despite programmatic interventions. Adolescent fertility did decline slightly from $89 / 1000$ to $87 / 1000$ between 2010 and 2014 [16], but this decrease is quite small and does not signify great success in efforts to substantially reduce early pregnancies as from 2011-2013, roughly 7\% of maternal deaths were accounted for by mothers between 15-19 [17]. Figure 1 depicts the drivers of teenage pregnancy in Swaziland.

Early pregnancy can have lasting negative effects in terms of sexual and reproductive health for young women and it poses high development costs for communities, particularly in perpetuating the cycle of poverty. In 2014, $16.7 \%$ of women between $20-24$ had at least one live birth before 18 years of age [5]. While this is a decline from 2010 , when $22 \%$ of young women reported having borne a child before 18 , the number of early pregnancies in Swaziland is still cause for concern [16].

\section{Contraceptive prevalence rate}

Contraceptive use is particularly important for the prevention of HIV/AIDS and other STIs and for the reduction of unintended and early pregnancies. Contraceptive prevalence (CPR) among all women aged $15-19$ years was $16.5 \%$ and among those aged $20-24$ was $60.2 \%$ [18]. In the general population, CPR has increased from $49 \%$ in 2007 to $65 \%$ in 2010 and slight increase to $67 \%$ in 2014 [5,6,16]. Among women aged 15-35, the most commonly reported contraceptives used were the male condom, injectable and female sterilization [19].

Women with little or no education report the lowest prevalence of contraceptive use or desired use, but also the highest proportion of unmet need. Similarly, women in lower age groups and wealth quintiles also face higher unmet need [15]. Essentially, the younger, poorer, and less educated woman in Swaziland is the more likely she is unable to access or utilize contraception. Figure 2 reveals the unmet among different wealth quintile and age.

\section{Youth, HIV/AIDS and other STIs}

A number of factors have been identified as the key drivers of HIV in Swaziland. These include multiple and concurrent sexual partnerships, low levels of male circumcision, early sexual initiation, late formation of stable relationships, low levels of consistent condom use, age-difference of partners in sexual relationships, income inequality, gender inequality and gender-based violence and mobility and migration [20].

\section{HIV incidence, prevalence and treatment}

Swaziland has the highest HIV prevalence in the world, and the epidemic begins to take a strong hold on the youth population during the early years of youth. Overall knowledge of drivers and prevention strategies for HIV among young people are low, with only $49.1 \%$ of women $15-24$ and $50.9 \%$ of men in the same age category able to correctly identify ways of preventing sexual transmission of HIV $[5,19]$. Knowledge of HIV status and both the prevalence and incidence rates of HIV within the youth population are also areas of concern. Many sexually active young people are not even aware of their HIV status. In $2014,80.2 \%$ of women aged $15-24$ who had been sexually active had been tested and knew their results. Only $62.3 \%$ of their male counterparts had been tested and knew their HIV status [21]. HIV testing is a critical first step in the continuum of care, and those who are HIV positive but no not know their results may be unknowingly transmitting the virus to their sexual partners (Figure 3).

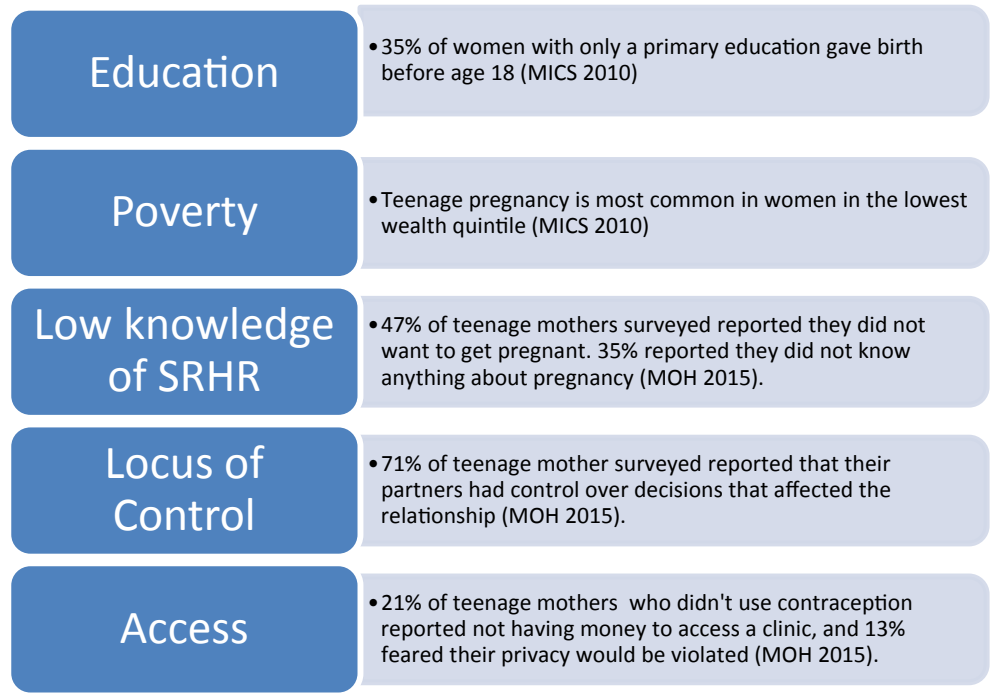

Figure 1: Selected drivers of teenage pregnancy in Swaziland. 

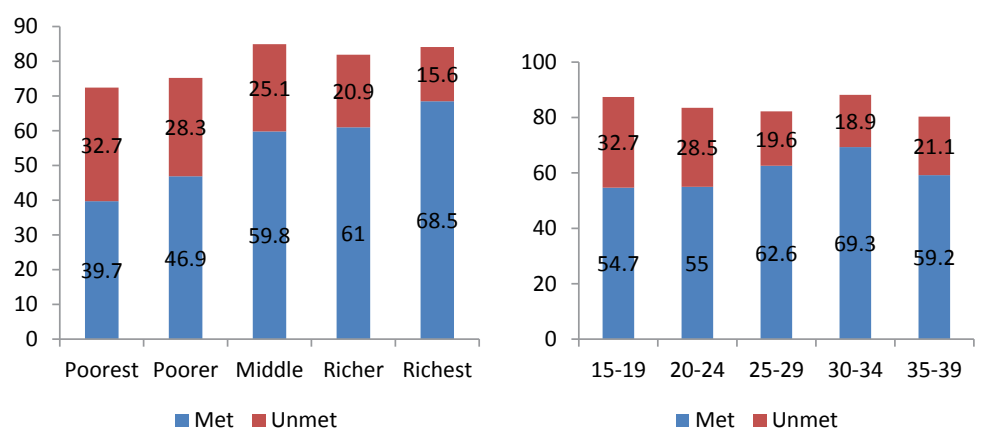

Source: Market segmentation analysis on family planning

Figure 2: Unmet need for family planning by wealth quintile and age.

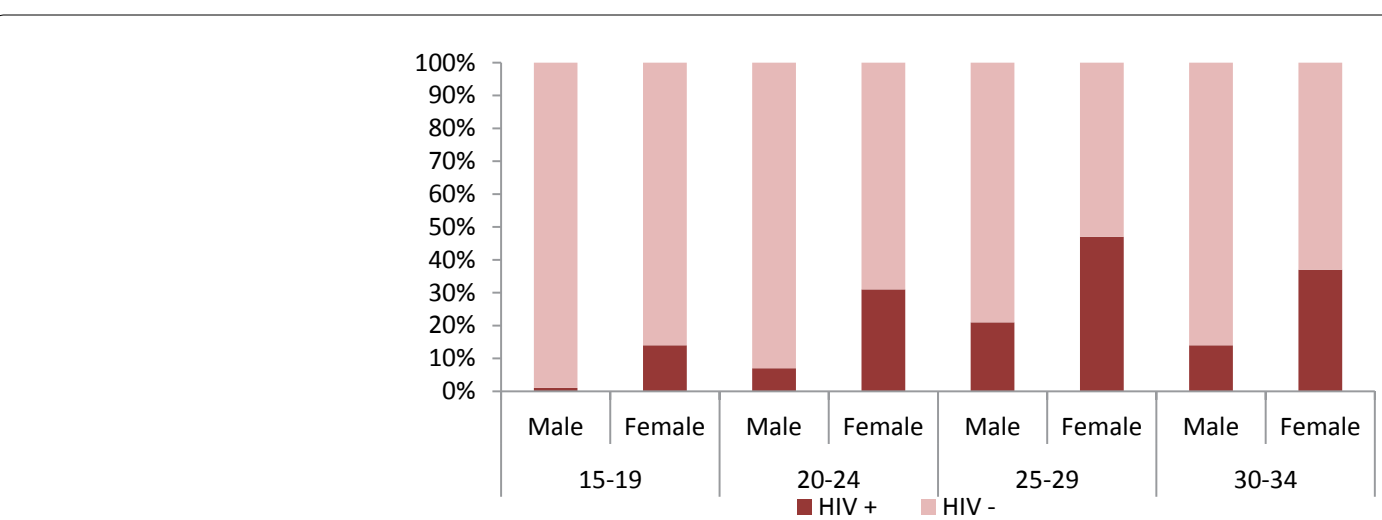

Source: Swaziland HIV incidence measurement survey, 2011

Figure 3: HIV prevalence among youth by age.

According to the 2006-07 SDHS the HIV prevalence in the total sexually active population was estimated at $26 \%$, while the incident rate was $2.9 \%$ [22]. A decline in the HIV incidence to $2.4 \%$ in the overall population was noted in 2011, but prevalence and incidence continue to be alarmingly high in the youth population [4].

HIV prevalence is much higher in young women than young men across all age groups, and marked increases in prevalence become apparent throughout the 20s for both sexes [4]. This implies that in future more effort should be directed to both young men and women, targeting them by age segmentation in order to effectively respond to HIV/AIDS. Figure 4 shows this discrepancy.

HIV incidence, or the rate of new infections, begins to accelerate rapidly in the youth population. For young women, an already high rate of new infections in the 15-19 age groups only increases in the early 20s [4]. For young men, incidence does not reach its climax until the early 30s, though HIV incidence in young men steadily increases from adolescence onward [4].

This data suggest that many new infections in young women are not due to sexual intercourse with young men in their own age bracket. Rather, young women are likely being infected by older men.

HIV treatment coverage for young people is not as high as it could be, particularly as emerging research continues to demonstrate the efficacy of treatment as a mechanism for preventing the transmission of new infections. Young people aged $15-19$ on ART was at $78 \%$ while the 20 -24years were at $77 \%$ in 2013 [21].

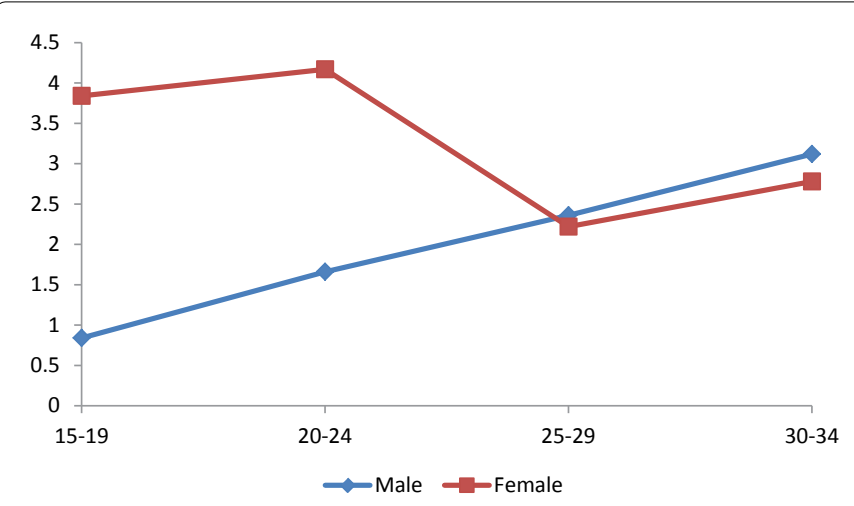

Source: Swaziland HIV Incidence measurement survey, 2011

Figure 4: New HIV incidence among youth.

\section{Sexual transmitted infections (STIs) other than HIV}

HIV/AIDS is a singular example of a particular SRH threat to the health of young people, but there are many other STIs to consider as well. Youth sexual behavior influences not only the acquisition of HIV, but of all other STIs, the presence of which makes youth more susceptible to HIV infection.

Drivers of STIs for young people can include multiple partners and the inconsistence or absence of condom use. Among sexually active young people $14-25,37.3 \%$ of women and $40.9 \%$ of men had engaged 
in sex with a non-regular partner, though reported condom use was higher, at $70.9 \%$ for young women and $93.4 \%$ for young men [16]. The diagnosis of STIs in Swaziland is based on symptoms present, rather than on screening, and since many STIs can be asymptomatic, the challenges of diagnosing and treating STIs extend beyond issues of access to SRH services for young people.

\section{Youth and gender-based violence}

Gender based violence is a public health concern and one of the critical areas of concern in Swaziland which profoundly affects young people - men, women and children. Approximately 1 in 4 females in Swaziland experienced physical violence as a child and $9 \%$ of the youth aged 18-24 experienced coerced sexual intercourse before they turned eighteen. Findings from a nationally representative sample of young women aged 18-24 in Swaziland indicated that 33\% had experienced sexual violence before the age of 18 , and that $24.4 \%$ of women $18-24$ had experienced sexual violence in the 12 months preceding the survey. Most incidents of such violence occurred either in the victim's own home or the home of a friend or neighbour. Between the ages of 18$24,32.6 \%$ of women report experiencing physical violence, and this violence is most often perpetrated by a boyfriend or husband, followed by other male relatives. Only $27.5 \%$ of respondents sought help of any kind [23]. Attitudes toward gender-based violence in general continue to be worryingly accepting, with $33.4 \%$ of women and $16.3 \%$ of men believe that a husband is justified in hitting his wife [16]. The majority of the respondents in agreement to this opinion were ages 15-24 years old, which shows the magnitude of the acceptability of gender-based violence within the youth population [19].

\section{Conclusion and Recommendations}

There seems to be some improvement in sexuality education and behaviour change interventions. There is gradual increase in age at sexual debut and in the proportion of adolescents reporting to be abstaining from sexual activity. This suggests that some interventions may have dissuaded some adolescents from sexual intercourse.

The likelihood of a young woman giving birth before 18 has decreased slightly, though early motherhood is still quite prevalent. A lower age at first birth is associated with education level attainment, wealth of the parents, and urban residence. Related to delayed fertility is also high use of contraception, especially for the peak years of the fertility curve for ages 25-30 years, though younger, poorer, and less educated women face more challenges in meeting their reproductive health needs. Public health facilities are the most popular methods of accessing contraceptives; however, they are not youth friendly. The efforts to provide youth friendly SRH services by organisations such as Family Life Association of Swaziland should be strengthened by integrating them with other services such as HIV and AIDS voluntary counselling and testing and STI.

There are high levels of gender based violence in Swaziland, affecting at least 1 in five women, and not exempting men. There are key policies and interventions within the context of gender and SRH that seek to address Gender Based Violence, such as: National Gender Policy 2010, the Child Protection and Welfare Act of 2012, the Sexual Offences and Domestic Violence Bill 2011, One Stop Centre, national surveillance system on violence, safe court system, and a number of interventions by government and civil society have been established.

\section{Key Recommendations for Sexual and Reproductive Health and Rights are below}

- Inter-ministerial government collaboration, in partnership with donors and civil societies, should strengthen public education on gender-based violence, HIV and SRH. The Ministry of Education and Training should mainstream LSE and gender in the curriculum at all levels including primary, secondary schools and tertiary and specialised colleges and vocational centres.

- A minimum standard for health facilities to be considered youth-friendly should be put in place, and all facilities should be assessed against the standard. The standard should address issues of accessibility of key SRHR and HIV services and commodities, as well as input from young people about the approachability of service providers.

- The availability of SRH and HIV information and services in schools should be strengthened, with appropriate linkages between schools, communities and health facilities as a key focus.

- The ability of young women to exercise control over their reproductive health should be strengthened by up scaling adolescent girls and young women's empowerment programmes and making a concerted effort to incorporate boys into programmes designed to empower girls and young women.

\section{Limitations of the Study}

The results of this retrospective study generated huge insights in regard to the HIV and Sexual Reproductive Health Status of Young People in Swaziland. However, the researchers of this study had no control over the primary data and or outcome assessment, as were reliant to secondary data. This was the key limitation of the study.

\section{Acknowledgement}

The authors are grateful to all the stakeholders who voluntarily participated in the report preparation.

\section{References}

1. United Nations Population Fund (2014) The power of 1.8 billion adolescents, youth and the transformation of the future: State of world population. UNFPA, New York.

2. United Nations Population Division (2013) World population prospects: 2012 revision. UNPD, New York.

3. Central Statistics Office (2007) Swaziland Population Projections 2007-2030. Government of the Kingdom of Swaziland, Mbabane.

4. Ministry of Health (2012) Swaziland HIV Incidence Measurement Survey. Mininstry of Health, Mbabane.

5. Central Statistics Office (2014) Multiple Indicator Cluster Survey Key Findings Government of the Kingdom of Swaziland, Mbabane.

6. Central Statistics Office (2010) Multiple Indicator Cluster Survey. Government of the Kingdom of Swaziland, Mbabane.

7. Ministry of health (2010) Service availability mapping report. Webster Print Mbabane.

8. Ministry of Health (2015) Risk Factors for Teenage Pregnancy and the Youth Perspective on Teenage Pregnancy and Health Needs in Nkalashane. Webster Print, Mbabane.

9. Ministry of Education and Training (2013) Education Management Information Systems: Annual Education Census Report. Ministry of Education and Training, Mbabane.

10. Ministry of Health (2013) National Policy on Sexual and Reproductive Health Government of the Kingdrom of Swaziland, Mbabane.

11. Ministry of Health (2016) Socio-cultural Factors Influencing Utilization of Sexua Reproductive Health Services Among Youth in Swaziland. Ministry of Health and UNFPA, Mbabane. 
Citation: Dlamini BR, Mabuza P, Masangane Z, Silindza T, Dlamini M, et al. (2017) The HIV and Sexual Reproductive Health Status of Young People in Swaziland: The Rationale for Focused Youth Investment. J AIDS Clin Res 8: 669. doi: 10.4172/2155-6113.1000669

Page 6 of 6

12. Ministry of health and Social Welfare (2007) National Health and Social Welfare Policy, Minstry of Health and Social Welfare, Mbabane.

13. Ministry of Sports (2009) Culture and Youth Affairs, National Youth Policy. Government of the Kingdom of Swaziland, Mbabane.

14. United Nations Children's Fund (2013) Situation Analysis of Children and Women in Swaziland. UNICEF, Mbabane.

15. Central Statistics Office (2012) Market Segmentation Analysis on Family Planning. CSO, Mbabane.

16. Central Statistics Office (2014) Multiple Indicator Cluster Survey 2010-2014 Comparative Report. Government of the Kingdom of Swaziland, Mbabane.

17. Ministry of Health (2015) Confidential Iquiry Report on Maternal Death Government of the Kingdom of Swaziland, Mbabane.
18. Central Statstics Office (2014) Multiple Indicator Cluster Survey. Government of the Kingdom of Swaziland, Mbabane.

19. Central Statistical Office, UNICEF (2016) Swaziland Multiple Indicator Cluster Survey 2014. Final Report, Mbabane.

20. National Emergency Response Council on HIVIAIDS (2014) Extended National Strategic Framework for HIVIAIDS 2014-2018. Government of the Kingdom of Swaziland, Mbabane.

21. Ministry of Health (2015) HIV Annual Report. Government of the Kingdom of Swaziland, Mbabane.

22. Central Statistics Office (2007) Demographic and Health Survey, Government of the Kingdom of Swaziland, Mbabane.

23. UNICEF Swaziland (2007) A National Study on Violence Against Children and Young Women in Swaziland. 